\title{
An Adaptive Algorithm to Optimize the Dynamics of IEEE 802.15.4 Networks
}

\author{
J. Hurtado-López, E. Casilari \\ University of Malaga, \\ ETSI Telecomunicacion, Campus de Teatinos, 29071 \\ \{jhurtado, ecasilari\}@uma.com
}

\begin{abstract}
IEEE 802.15.4 standard is becoming one of the most popular technologies for the deployment of low rate Wireless Personal Area Networks with strong power constraints. In order to reduce the energy consumption, beacon-enabled networks with long network inactive periods can be employed. However, the duration of these inactivity periods, as some other configuration parameters, are conventionally set to default values and remain fixed during the whole network operation. This implies that if they are misconfigured the network will not adapt to changes in the conditions of the environment, particularly to the most determining one, i.e. the traffic load. This paper proposes a simple procedure for the dynamic adaptation of several key parameters of IEEE 802.15.4 networks. Under this procedure, the 802.15.4 parameters are modified as a function of the existing traffic conditions.
\end{abstract}

Keywords: IEEE 802.15.4, Wireless Sensor Networks, optimization, CSMA/CA

\section{Introduction}

IEEE 802.15.4 standard [1] defines the Physical layer (PHY) and the Medium Access Control layer (MAC) for the communication of low-power Wireless Sensor Networks (WSN). Specifications such as ZigBee [2] or 6LoWPAN [3] are built on IEEE 802.15.4 standard to complete the protocol stack for Low-Rate Wireless Personal Area Networks (LR-WPAN). This stack is designed to satisfy the market needs for energy efficient, low cost (bellow one dollar) and low rate wireless embedded devices. IEEE 802.15.4 compliant transceivers operate in the Industrial Scientific and Medical (ISM) radio bands with a maximum transfer rate of $250 \mathrm{kbps}$ at $2.4 \mathrm{GHz}$ (with 16 available channels), which can be decreased to $40 \mathrm{kbps}$ or even down to $20 \mathrm{kbps}$ at the $915 / 868 \mathrm{MHz}$ bands (channels 0 to 10). The standard also contemplates the possibility of providing real time services through Guaranteed Time Slots (GTS).

There are two different modes for the MAC sublayer to operate: (1) the beaconless mode, also denominated point to point, in which unslotted CSMA/CA is used between nodes to communicate, and (2) the beacon-enabled mode, which utilizes slotted CSMA/CA. In this last case communications are synchronized through the transmission of beacons, i.e. a special type of frame that is periodically emitted by specific nodes (coordinators). In order to keep synchronized, nodes must associate to 
a coordinator and stay active to receive the Beacon. Under this beacon mode, transmissions are only allowed within a special period, the Contention Access Period (CAP), which begins immediately after the Beacon emission and whose duration is defined by the coordinator. After the CAP, GTS (Guaranteed Time Slots) may take place. During the remaining time until the next Beacon, the nodes enter into a low consumption state (or sleeping mode) reducing their duty cycle and consequently saving battery power. Although the beaconless operation mode is less complex and does not present any scalability problem (as far as it allows nodes to transmit at any moment), it may force the nodes to be listening to the radio channel continuously. This leads to a useless waste of energy while GTS are not possible. On the other hand, the beacon-enabled mode is more complex to configure and implement as it may demand a strict synchronization of the nodes.

The main challenge and also the main attractiveness of IEEE 802.15.4 is its potentiality to set up self-organizing networks capable of adapting to diverse topologies, node connectivity and traffic conditions. In fact, most advantages of employing IEEE 802.15.4 strongly depend on the configuration of the Medium Access Control (MAC) sublayer.

This paper proposes several enhancements for the dynamics of the IEEE 802.15.4 MAC layer. The proposal includes different algorithms to adapt and optimize the activity periods and the time of transmission of the nodes in an IEEE 802.15.4 compliant star network according to the traffic load. The analysis of the performed simulations shows that a wrong election of the beacon-enabled mode parameters may severely affect the global network behavior.

This paper is organized as follows: Section 2 briefly describes the configuration and operation of beacon-enabled IEEE 802.15.4 network. The section also reviews some existing proposals to adapt the configuration to the traffic load. Section 3 presents the algorithms proposed to optimize the network performance while Section 4 compares them by means of simulations. The final Section 5 summarizes the main conclusions and suggests some possible research lines.

\section{Configuration of 802.15.4 Networks}

The IEEE 802.15.4 standard defines two types of devices: Full-Function Devices (FFD) and Reduced-Function Devices (RFD). The last ones are only enabled to communicate with its coordinator. Typical leaf nodes, such as sensors, will be RFDs. FFDs may play any role in the network, i.e. coordinator (PAN coordinator or intermediate router in multihop networks) or leaf node. A coordinator manages and centralizes the communications of a star topology formed by a set of associated nodes. When operating in a beacon-enabled mode a coordinator announces itself and the corresponding network identifier by broadcasting beacons periodically. The nodes associated with a coordinator must synchronize to this frame. The time between two consecutive beacons is called the Beacon Interval $(B I)$ and its structure is called Superframe (see Figure 1). The Superframe can be divided into two periods: an active part and an inactive one. All the communications between a coordinator and its 'children' must take place during the active portion of the Superframe, also known as 
Superframe Duration $(S D)$. All nodes, including the coordinator may go into a power saving mode or sleeping state during the inactive period to extend their batteries lifetime.

The whole structure of the Superframe is governed by the values of two MAC numerical parameters: the macBeaconOrder $(B O)$ and the macSuperframeOrder $(S O)$. $B O$ and $S O$ define the values of $B I$ and $S D$ as it follows:

$$
\begin{gathered}
B I=a \cdot 2^{B O} \text { for } 0 \leq B O \leq 14 \\
S D=a \cdot 2^{S O} \text { with } 0 \leq S O \leq B O \leq 14
\end{gathered}
$$

where $a$ is the Base Superframe duration (15.36, 24 or $48 \mathrm{~ms}$ depending on the employed bit rate: 250,40 or $20 \mathrm{kbps}$ respectively). The values of $B O$ and $S O$ are limited to the $[0,14]$ interval. In addition the value of the $S O$ must remain equal or lower than $B O$. The ratio $S O / B O$ is called the duty-cycle. The lower the duty-cycle the larger the inactive period. If $S O=B O$ (i.e. duty-cycle is 1 ) no inactive period would exist and the Superframe Duration would coincide with the whole Beacon Interval.

The active period of the superframe is divided into sixteen slots. The first one (slot 0 ) is reserved for the beacon. This frame must be received by all the associated devices so that they must be awake for this first slot. Up to seven Guaranteed Time Slots may be assigned to some nodes at the end of the $S D$ in order to provide QoS (Quality of Service). This is called the Contention Free Period (CFP). The Contention Access Period (CAP) extends between slot zero and the CFP. Within the CAP, devices contend for the channel and communications are regulated by slotted CSMA/CA.

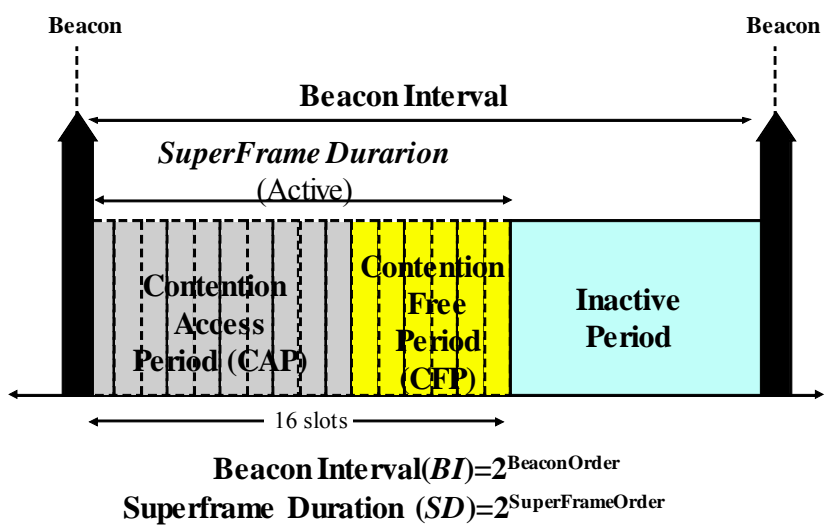

Fig. 1. 802.15.4. Superframe.

\subsection{CSMA/CA}

Slotted CSMA/CA channel access algorithm shall be normally used in IEEE 802.15.4 beacon-enabled networks to transmit data or commands within the CAP. Figure 2 illustrates the Slotted CSMA/CA algorithm flow chart. 


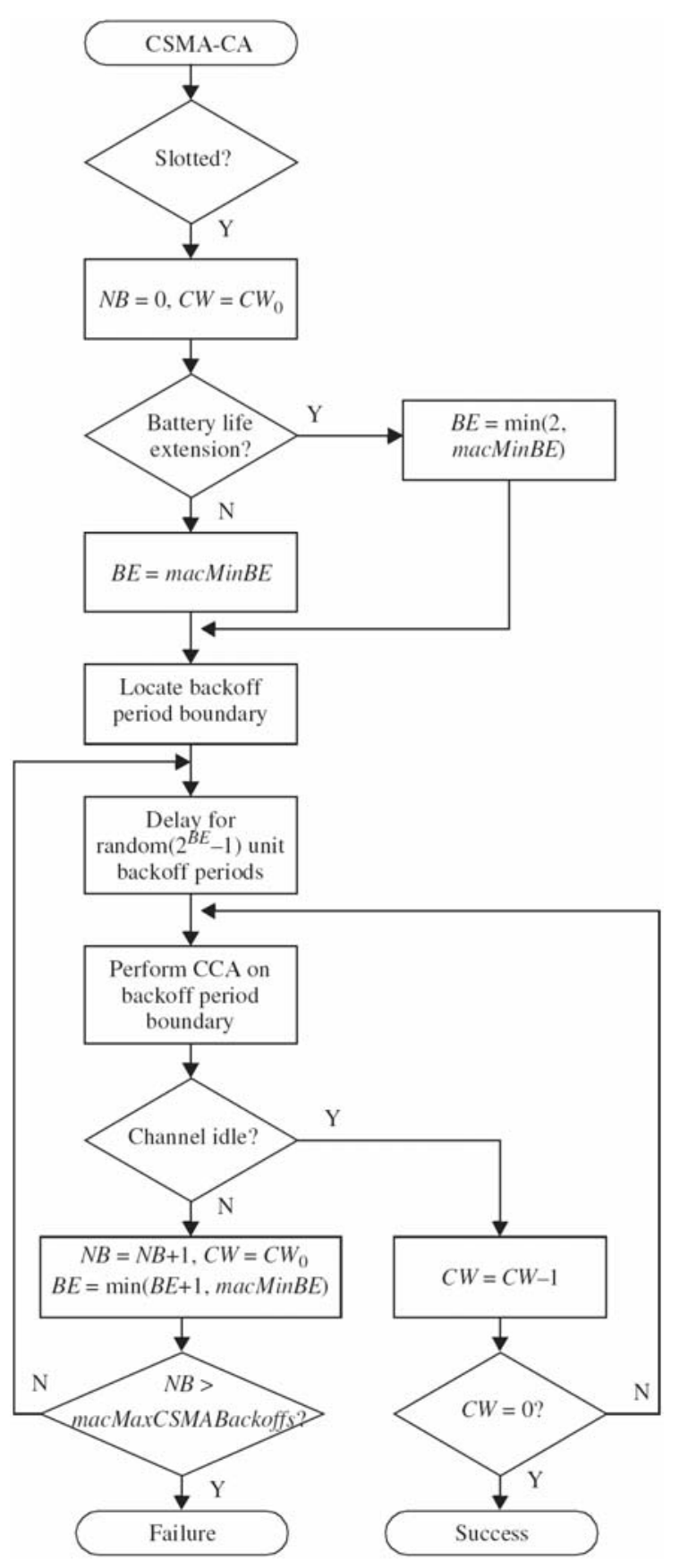

Fig. 2. Stotted CSMA/CA algorithm [1] 
The algorithm is implemented using its own unit of time called the backoff period (time required to transmit 20 symbols: $320 \mu$ s when operating in the $2.4 \mathrm{GHz}$ band). A collision occurs when two or more transmissions take place at the same time. If this happens data are lost and network performance degrades. Aiming at avoiding collisions CSMA/CA follows two different strategies: firstly, nodes must wait for a random number of backoff periods before trying to transmit and secondly the channel is sensed to detect activity. Each transmission attempt is controlled by three variables: $N B, C W$ and $B E . N B$ stores the number of times that the current transmission has been attempted. It is initialized to zero before each transmission and incremented in one unit if the channel is sensed to be busy. If $N B$ rises above the threshold defined by macMaxCSMABackoffs, transmission is aborted and the algorithm terminates with a channel access failure status. $C W$ is initialized to two (except for the $950 \mathrm{MHz}$ Japanese band) and defines the number of backoff periods that the channel has to be consecutively sensed idle before a transmission. If the channel is detected to be busy, $\mathrm{CW}$ is reset to zero. The parameter $B E$ controls the number of random backoff periods (in the range from 0 to $2^{\mathrm{BE}}$ ) that the nodes must wait before proceeding to the channel assessment. If the binary configuration parameter Battery Life Extension (BLE) is set to false (zero) $B E$ shall be initialized to the value of macMinBE. Otherwise, if true, $B E$ shall be initialized to the lesser of 2 and the value of macMinBE. If the channel is assessed to be busy, $B E$ shall be set to the minimum of $B E$ incremented by 1 and $\operatorname{macMaxBE}$.

\subsection{Related works}

Most of the papers studying the slotted CSMA/CA algorithm employ a Markov chain model for their analysis [4] [5] [6]. The paper in [4] analyzes and simulates the throughput and energy consumption of a 802.15.4 network under saturated traffic conditions and for a different number of nodes. This study shows that, as the number of nodes increases, the throughput decreases while the energy consumption per one slot payload increases. An extension to non-saturated traffic conditions can be found in [5]. Their analysis probes that for saturated networks, it is preferable to choose a large exponential delay backoff in order to reduce the required energy per useful bit. On the other hand, for unsaturated networks, a very small energy saving can be achieved by setting smaller backoff values. In [6] the authors characterize (also using the theory of discrete time Markov chains) the interaction of multiple parameters such as the packet arrival rate, the number of nodes, the queue length, the packet size and the Beacon Interval. They conclude that the size of the network must be kept very small in order to maintain the mean packet service time below the duration of the superframe.

As it refers to those works that study the MAC layer performance for different values of its configuration parameters (e.g. macMinBE, macMaxFrameRetries, macMaxCSMABackoffs), those in [7], [8], and [9] should be highlighted. In [7] and [8], the authors evaluate by simulation and experimentation the reliability of the MAC layer. They conclude that, when using the default values proposed by the standard, as the number of network nodes increases, the rate of delivery or delivery ratio, rapidly decreases. This performance decline is shown to be significant even for only five 
network nodes. In [9] a cross-layer solution to the various problems encountered is proposed.

Aiming at maximizing the throughput for a beaconed star topology the article in [10] focuses on the impact of the Backoff Exponent $(B E)$. According to their ns-2 simulations the authors state that reducing the minimum default value of $B E$ from three to one and adjusting it individually for every node (basing on the data to be transmitted) can result in an increase in the transfer rate of up to $45 \%$. On the other hand, in the slotted version of CSMA/CA, if it is not possible to transmit in the a certain CAP, the packets are stored by the nodes so that their transmission is deferred until the beginning of the next CAP. This causes transmission attempts to be concentrated at the beginning of the CAP, which results in an increment in the packet collision probability. This is known as the access congestion problem. The more the network is saturated the more this problem will arise. The origin of the access congestion problem according to [11] is that, to reduce the consumption of the network, the initial value of $\mathrm{BE}$ is too small. This provokes that many nodes will calculate the same random delay value and consequently they will try to transmit simultaneously, which will induce packet collisions. The authors propose an algorithm that adapts the value of BE to the particular circumstances of the network to alleviate the problem. Authors suggest that the same algorithm may be applied to check the network load and to adapt the superframe duration appropriately to fit the requirements of the network under the current traffic load. However, the proposal is left for further research.

The main way to adapt a beaconed 802.15.4 network to the traffic conditions is to modify the duty-cycle, i.e. the ratio between the Superframe Order and the Beacon Order. A traffic adaptive Superframe Order is proposed in [12]. In this work if the queue occupation of a node exceeds a certain threshold it issues a special packet to the coordinator. Once that it is received, the coordinator sets a $100 \%$ duty cycle in the next superframe. When the coordinator does not receive any of these packets during several superframes it diminishes the value of the Superframe Order. The weakness of this approach is that it requires that the node can communicate with the coordinator. Thus if the node's queue is full because of the device's difficulties to transmit, the coordinator will not receive the packet and no adaption will be performed to the SO. A possible solution can be found in [13] where a special broadcast tone is emitted by a node after the CAP if it is unable to transmit or have not received the acknowledgement packets. The coordinator extends the CAP when it receives this tone. However this idea is not fully compatible with the standard as it requires the coordinator to be active after the CAP. Moreover it cannot be easily extendable to clustered networks. A different approach can be found in [14] and [15] where the coordinator is in charge of estimating the need of adaption by tracing the frequency of the communications of its children. In these papers the same duty-cycle adaptation to traffic can be achieved by modifying the Beacon Order parameter instead of the Superframe Order. The main conclusion of these studies is that certain trade-offs between the desired power saving and the delay have to be found. Energy can be saved if delay is sacrificed.

Our study is focused in relatively large networks (101 nodes) under heavy traffic conditions. 


\section{Proposed Algorithms}

In the following subsection we present two strategies that are intended to reduce the impact of a bad election of the Superframe Order (SO) and the initial random CSMA/CA backoff wait time on the performance of a 802.15.4 star network.

\subsection{Superframe Order adaptation algorithm}

As it can be observed in expressions (1) and (2), for beacon-enabled networks, both $B O$ and $S O$ are key parameters. It is important to notice that they are defined as constant parameters. Thus, once they are configured, their value will never change regardless of the circumstances of the network (in particular, the traffic load). It may occur that real traffic network conditions or traffic patterns differ from those assumed during the design and the deployment of the network leading to a performance degradation which will be caused by a misconfiguration of these parameters.

The time between two consecutive Beacons i.e. the Beacon Interval, only depends on the $B O$ parameter, therefore it seems reasonable that, in a star network, the whole average latency strongly depends on the $B O$ selection. This is why the $B O$ value is typically dictated by the application level and the actual requirements of the corresponding WSN. For this reason, in this paper we do not consider the adaptation of the $B O$. Thus, regarding the SuperFrame Structure only the $S O$ parameter can be modified in order to try to accommodate the network configuration to the traffic.

Selecting a wrong Superframe Order value can have serious implications for the performance of the network even if the traffic is always the same. If the value configured for the $S O$ is low and the traffic is high, the network will most probably not be able to process all the packets properly since contention access periods will be too small. On the other hand, suppose the extreme case of a network in which the SuperFrame Order is set to a high value and there is no traffic; this configuration would unnecessarily force the coordinator to be active during long periods, increasing the consumption and reducing the battery lifetime. This fact is aggravated in the case of cluster multihop networks where more nodes acting as intermediate routers exist.

A different scenario could be that of a network where traffic conditions vary. For example, consider a WSN in which several types of sensors coexist. Suppose that most of the time there is little or no traffic, in this case it seems appropriate to fix the value of the $S O$ to its minimum, i.e. zero. However it may happen that some of the sensors, according to their nature, periodically turn on and transmit data causing local traffic peaks. In this scenario it would be adequate to dynamically adapt the value of $S O$, increasing it when those sensors activate and returning to the minimum once the traffic is processed. So, the main goal of our adaptive scheme consists in trying to detect the local variations that may occur in the traffic and then decide whether the $S O$ should be changed or not.

In our solution, as a first strategy, we propose that the network coordinator performs a count of the number of frames received from each of its children at every interval between beacons and computes the relative increase or decrease of the traffic load. Then there are three possibilities. Firstly, if an important traffic growth is detected the coordinator will assume that the traffic has considerably increased so that 
the duty cycle of the nodes ( $\mathrm{SO} / \mathrm{BO}$ ratio) should be augmented. In that case, the value of $S O$ is incremented in 1 unit in order to enlarge the Superframe Duration and to make more time available for data transmissions. Secondly, if a significant reduction is computed, traffic is considered to have decreased and $S O$ should be decremented (if possible). If not relevant changes in the traffic load are detected, the Superframe Order is left unchanged.

The modification of the Superframe Order in a Start Network is extremely easy to propagate to the whole network as it only involves the transmission of the new value of the $S O$ in the next Beacon (every Beacon contains a reserved field to inform about the value of the $S O$ ) and every node must listen to the coordinator's Beacons. So this algorithm is fully compatible with the standard and does not introduce any protocol overload in the sense that it requires no additional information. In our implementation we have included two different control parameters so that two thresholds $\left(U_{1}, U_{2}\right)$ can be set to determine if a change in the relative traffic received from a node is significant or not. The first one $\left(U_{l}\right)$ determines when the $S O$ must be increased while the second one $\left(U_{2}\right)$ governs the reductions of the superframe order.

Mathematically the adaptation of $S O$ can be described as:

$$
\begin{aligned}
& \left\{\begin{array}{l}
{[x[n+1]-x[n]]>U_{1}} \\
\text { and } \\
S O[n+1]<B O
\end{array}\right\} \Rightarrow S O[n+2]=S O[n+1]+1 \\
& \left\{\begin{array}{l}
{[x[n+1]-x[n]]<-U_{2}} \\
\text { and } \\
S O[n+1]>0
\end{array}\right\} \Rightarrow S O[n+2]=S O[n+1]-1
\end{aligned}
$$

where $x[n]$ and $S O[n]$ respectively represents the traffic (number of packets) received by the coordinator and the value of the Superframe Order during the $n$-th beacon interval, while and $U_{l}$ and $U_{2}$ are the aforementioned decision thresholds.

\subsection{Backoff Exponent adaptation algorithm}

This is the second adaptation strategy presented in this paper. As seen in the section 2.1 the Backoff Exponent $(B E)$ parameter is another fundamental parameter in the CSMA/CA algorithm. Depending on its value a node determines the random delay time before every transmission to minimize the probability of collision. By default, 802.15.4 standard establishes an initial value of three for $B E$, which can be incremented up to five if the radio channel is sensed to be busy twice. Even if $B E$ is 5, just a maximum of thirty-one different backoff waiting periods are possible, while a CAP enables up to 786,432 backoff periods. So it is not unusual that some nodes will calculate the same random delay value and try to transmit simultaneously causing a collision. This may become a major problem for those configurations of star networks in which the CAP is not large enough to process all the traffic load or if there is a high traffic density within the active Superframe, which may be caused by a 
misconfiguration of the $S O$ parameter. Another related problem is the access congestion which was previously described.

As a solution to alleviate these problems we propose an algorithm that adapts the value of $B E$ to the particular circumstances of the network. The idea behind the algorithm is to calculate the random delay time that precedes every transmission following a uniform distribution along a configurable percentage of the overall duration of the Contention Access Period. By increasing the random wait before the packet emission, the algorithm aspires to reduce the Access Congestion Problem with a better distribution of the traffic within the CAP. This is obviously achieved at the cost of increasing the packet delay.

\section{Simulation and Results}

In order to evaluate the performance of the precedent algorithms, we have implemented and simulated them in the OMNeT++ 4.2.2 Inetmanet IEEE 802.15.4 UndertTest environment [16], [17]. The selected scenario consists of a star network topology formed by a hundred leaf nodes and a coordinator. Neither the network creation phase nor Guaranteed Time Slots are considered. There is no possible hidden node effect because every node is in the range of interference of the rest. In our study there only exists uplink traffic, i.e. from the nodes to the coordinator, except for the Beacons. This could be a realistic scenario of a wireless sensor network in which the sensors (leaf nodes) consist of simple RFD end devices while the coordinator could be a more complex FFD node acting as a sink of the information sent by the sensors.

The network is programmed to operate at the channel $11(2.4 \mathrm{GHz}$ band $)$ at 250 kbps. As the performance metrics we define:

-Queue Drops: percentage of packets discarded by nodes' queue. A node queue drops a packet when it is full. The selected queue length is 10 packets.

-Transmission losses, which reflect the percentage of packet transmissions that have reached the maximum number of allowed retries so that the packet is dropped by the node.

-Average delay: for every packet received by the coordinator we compute the difference between its generation and arrival times. Thus the average delay in seconds is defined as the average of all these differences.

-Collisions: The overall number of collisions that take place in the network.

-Energy per bit: global cost (miliJoules) involving the transmission of a single bit, defined as:

$$
\text { Energy/bit }\left(\frac{\mathrm{mJ}}{\text { bit }}\right)=\frac{\text { Network Consumed Energy }(\mathrm{W} \cdot \mathrm{s})}{(\text { Total Received Bytes }) \times 8} \times 1000
$$

For all the simulations the Beacon Order is set to 5 and the packet size is 10 bytes while the inter arrival time of the packets follow an exponential distribution with a mean value of $1 \mathrm{~s}$. For most typical applications of WSN, this configuration of the network (100 leaf nodes and one packet per second and node) can be considered an example of heavy traffic load conditions as long as an average of 100 packets will be sent to the coordinator every second. Most of these packets will contend for the same 
radio resource during the CAP. Thus many collisions, delays and packets drops are expected to occur.

The employed energy model storages the time a device stays in each of the four possible states idle, reception, sleep and transmission in seconds. Table 1 presents the current consumption in $\mathrm{mA}$ for a typical 802.15.4 device [18].

The device energy consumption $(\mathrm{mW} \cdot \mathrm{s})$ for each state can be easily computed as the product of $\mathrm{Vcc}(\mathrm{V})$, the current consumption $(\mathrm{mA})$ and the time the node expends in that state (s).

Table 2 tabulates the reference results obtained after the simulation of the network when no adaptive policy is applied.

Table 1. Consumption of a device depending on the status of the radio transceiver for a supply voltage $\left(\mathrm{V}_{\mathrm{CC}}\right)$ of $3.3 \mathrm{~V}[18]$

\begin{tabular}{ll}
\hline Status & Consumption $(\mathrm{mA})$ \\
\hline Idle & 0.42 \\
Reception & 19.70 \\
Sleep & 0.02 \\
Transmission $(0 \mathrm{dBm})$ & 17.40 \\
\hline
\end{tabular}

Table 2. Reference results

\begin{tabular}{cccccc}
\hline SO & $\begin{array}{c}\text { Queue Drops } \\
\text { (\%) }\end{array}$ & $\begin{array}{c}\text { Transmission } \\
\text { Loss (\%) }\end{array}$ & $\begin{array}{c}\text { Average } \\
\text { Delay (s) }\end{array}$ & $\begin{array}{c}\text { No. of } \\
\text { Collisions }\end{array}$ & $\begin{array}{c}\text { Energy/bit } \\
\text { (mJ/bit) }\end{array}$ \\
\hline $\mathbf{0}$ & 89.28 & 66.29 & 16.256 & $6,408,421$ & 0.759 \\
$\mathbf{1}$ & 83.28 & 33.20 & 1.561 & $11,008,155$ & 0.309 \\
$\mathbf{2}$ & 71.96 & 24.67 & 0.407 & $15,749,402$ & 0.194 \\
$\mathbf{3}$ & 47.19 & 34.36 & 0.224 & $15,760,231$ & 0.118 \\
$\mathbf{4}$ & 0.00 & 18.31 & 0.089 & $2,467,858$ & 0.021 \\
\hline
\end{tabular}

Note that the traffic generated by nodes is the same for every simulation, so if we observe the evolution for the queue drops as the $S O$ value increments we can see a logical decrease. We should take into account that a unit increase in the value of the $S O$ means duplicating the duration of the CAP so that the probability of successfully transmitting a packet and releasing a queue position significantly increases. When $S O$ is bigger than three, the coordinator's Superframe Duration is long enough to accommodate all the traffic transmitted by the nodes so that there are no queue drops. On the other hand, for lower $S O$ values the CAP is too short, the nodes have to store the packets and the queue begins to reach its full capacity at some instants (dropping some packets). A misconfiguration of the Superframe Order to zero will cause serious saturation problems, with loss rates higher than $80 \%$. The same evolution with the $S O$ is followed by the Average Delay. A $B O$ of 5 corresponds to a Beacon Interval of approximately half a second $(0.49152 \mathrm{~s})$. As it can be noticed from Table 1 , the average delay for $S O$ values over two is below a Beacon Interval, which is a desired property. If $S O$ equals to 0 the average delay exceeds more than one order of magnitude the duration of a Beacon Interval, which will most probably be not tolerable. 
The relationship between collisions and the Superframe Order is not so simple. In fact, if we compare with the case with $S O=0$, we can observe that collisions initially increase when a higher $S O$ is utilized. The reason for this trend is that if $S O$ is zero, the CAP is too short even to try to transmit most packets, which are directly dropped without provoking any collision. In this sense, just for the longest CAP $(S O=4)$, the collisions tend to decrease.

Table 3 reflects the main results obtained after the simulation of the first policy. The values for $U_{l}$ and $U_{2}$ were heuristically set to 20 and 70, respectively.

Table 3. Results with Superframe Order Adaptation.

\begin{tabular}{ccccc}
\hline $\begin{array}{c}\text { Queue Drops } \\
\text { (\%) }\end{array}$ & $\begin{array}{c}\text { Transmission } \\
\text { Losses (\%) }\end{array}$ & $\begin{array}{c}\text { Average } \\
\text { Delay (s) }\end{array}$ & $\begin{array}{c}\text { Global } \\
\text { Collisions }\end{array}$ & $\begin{array}{c}\text { Energy/bit } \\
\text { (mJ/bit) }\end{array}$ \\
\hline 72.08 & 15.34 & 0.274 & $13,981,844$ & 0.153 \\
\hline
\end{tabular}

The results show that the Superframe Order adaptation algorithm presents a good behavior. The average delay remains under the Beacon Interval while the transmission fail rate is lower than any of the reference values. However this policy does not reduce the number of collisions, mainly caused by the heavy traffic conditions existing during the initial phase of the CAPs. This problem can be mitigated by ignoring the initial default value of $B E$. Table 3 shows the results of the reference experiments when the Backoff Exponent Policy is utilized. In this case, before any transmission, a random waiting time between 0 and a percentage of the remaining CAP is selected. As it is shown in Table 4, under this policy both collisions and transmission losses are mitigated while more traffic is transmitted for any value of $S O$ (see Table 5).

Table 4. Results with the Backoff Exponent adaptation Policy.

\begin{tabular}{cccccc}
\hline SO & $\begin{array}{c}\text { Queue Drops } \\
\text { (\%) }\end{array}$ & $\begin{array}{c}\text { Transmission } \\
\text { Loss (\%) }\end{array}$ & $\begin{array}{c}\text { Average } \\
\text { Delay (s) }\end{array}$ & $\begin{array}{c}\text { Global } \\
\text { Collisions }\end{array}$ & $\begin{array}{c}\text { Energy/bit } \\
\text { (mJ/bit) }\end{array}$ \\
\hline $\mathbf{0}$ & 87.44 & 63.24 & 39.089 & $5,319,702$ & 0.474 \\
$\mathbf{1}$ & 78.33 & 40.69 & 27.762 & $9,299,981$ & 0.251 \\
$\mathbf{2}$ & 60.28 & 26.58 & 19.208 & $11,926,301$ & 0.155 \\
$\mathbf{3}$ & 21.59 & 16.81 & 9.479 & $9,007,508$ & 0.116 \\
$\mathbf{4}$ & 0.00 & 1.05 & 0.436 & 803,265 & 0.022 \\
\hline
\end{tabular}

Table 5. Improvement of the transmitted traffic under Backoff Exponent adaptation Policy.

\begin{tabular}{cc}
\hline SO & Increment of transmitted Bytes (\%) \\
\hline $\mathbf{0}$ & 1.00 \\
$\mathbf{1}$ & 1.63 \\
$\mathbf{2}$ & 8.10 \\
$\mathbf{3}$ & 30.56 \\
$\mathbf{4}$ & 17.27 \\
\hline
\end{tabular}

Furthermore, the energy per bit is kept within very reasonable values improving the reference ones in practically all cases. The main disadvantage of this technique is the delay, which is substantially increased. This was expected since the technique postpones the transmission of the packets increasing the random average waiting 
time. The percentage of the CAP employed by the algorithm to calculate the random waiting time prior to each transmission is $100 \%$ in this paper.

Finally Table 6 collects the results obtained if both policies are simultaneously applied.

Table 6. Results of the combined policy

\begin{tabular}{ccccc}
\hline $\begin{array}{c}\text { Queue } \\
\text { Drops (\%) }\end{array}$ & $\begin{array}{c}\text { Transmission } \\
\text { Fails (\%) }\end{array}$ & $\begin{array}{c}\text { Average } \\
\text { Delay (s) }\end{array}$ & $\begin{array}{c}\text { Global } \\
\text { Collisions }\end{array}$ & $\begin{array}{c}\text { Energy/bit } \\
\text { (mJ/bit) }\end{array}$ \\
\hline 0.00 & 1.48 & 0.436 & 797,991 & 0.022 \\
\hline
\end{tabular}

The combination of both techniques yield reasonable values for the queue losses, transmission fails and the energy per bit while the average delay is still smaller than a beacon interval. Furthermore the number of collisions has noticeably decreased and it improves all previous results. Also, the increase in the number of transferred bytes is of $172.11 \%$.

If none of the presented techniques is applied the best record for the transmission losses $(18.31 \%)$ is reached for a value of $S O=4$. A slightly better result is obtained with the Superframe Order Adaptation policy with 15.75\%. However, after combining the two techniques the obtained value plummets down to $1.05 \%$.

\section{Conclusions and Future Work}

This paper has investigated the dynamic optimization of two key IEEE 802.15.4 MAC sublayer parameters, the Superframe Order, and the Backoff Exponent. The Standard defines the Superframe Order parameter but does not mention how to determine it. Furthermore, $S O$ is defined as a constant. We have shown that $S O$ value has a deep impact on the network performance so we propose its dynamic adaptation to the network conditions, particularly to the traffic load. In this regard it has been proposed, implemented and simulated a technique which adapts the size of the Contention Access Period to the actual traffic load by reconfiguring the $S O$. We have also studied the collisions and access congestion problem and presented a policy to avoid them. One of the most promising features of IEEE 802.15.4 networks is its capability for self-configuring. This is why we consider that adaptive policies can be of great interest for the optimization of 802.15.4 networking applications. Finally, we have presented the most significant results obtained by the simulation of the proposed techniques when they are applied both separately and jointly being particularly. Results show that the combination of both techniques leads to a better network performance. Future work should extend these studies to the cluster-tree topologies where problems as the Access Congestion will become even more important. Additionally, we propose the study of other policies for network reconfiguration where the adaptability to traffic should be managed in the end (leaf) nodes. 


\section{Acknowledgments}

This work was partially supported with public funds by the Spanish National Project No.TEC2009-13763-C02-01.

\section{References}

1. IEEE-WG802.15, "802.15.4-2011 - IEEE Standard for Local and metropolitan area networks--Part 15.4: Low-Rate Wireless Personal Area Networks (LR-WPANs)", IEEE standard for Information Technology, 2011.

2. ZigBee-Alliance, (2013). "ZigBee specification", Available: http://www.zigbee.org/

3. N. Kushalnagar et al., "IPv6 over Low-Power Wireless Personal Area Networks (6LoWPANs): Overview, Assumptions, Problem Statement, and Goals”, RFC. 4919, Internet Engineering Task Force (IETF), August 2007.

4. T.R. Park et al., "Throughput and energy consumption analysis of IEEE 802.15.4 slotted CSMA/CA", Electronics Letters, September 2005.

5. S. Pollin et al., "Performance Analysis of Slotted Carrier Sense IEEE 802.15.4 Medium Access Layer", IEEE Trans. on Wireless Communication, 7:9,(3359-3371), September 2008.

6. J. Mišic et al., "The impact of MAC parameters on the performance of 802.15.4 PAN,"Ad Hoc Netw., vol. 3, no. 5, pp. 509-528,September 2005.

7. G. Anastasi et al., "The MAC unreliability problem in IEEE 802.15.4 wireless sensor networks" Proceedings of the 12th ACM international conference on Modeling, analysis and simulation of wireless and mobile systems, pp. 196-203, ACM, Spain, 2009

8. G. Anastasi et al., "A Comprehensive Analysis of the MAC Unreliability Problem in IEEE 802.15.4 Wireless Sensor Networks," IEEE Trans. Industrial Informatics, vol.7, no.1, pp.52,65, February 2011. doi: 10.1109/TII.2010.2085440

9. M. Di Francesco et al., "Reliability and Energy-Efficiency in IEEE 802.15.4/ZigBee Sensor Networks:An Adaptive and Cross-Layer Approach," IEEE J. Sel. Areas Commun., vol.29, no.8, pp.1508-1524, September 2011

10. J. G. Ko et al., "Performance Evaluation of IEEE 802.15.4 MAC with Different Backoff Ranges in Wireless Sensor Networks", Proceedings of the 10th IEEE International Conference on Communication systems (ICSS), pp. 1-5, Singapore, October 2006,

11. V. Rao and D. Marandin, "Adaptive Backoff Exponent Algorithm for Zigbee (IEEE 802.15.4)", Next Generation Teletraffic and Wired/Wireless Advanced Networking, Vol. 4003, pp. 501-516, Germany 2006. surpassed

12. Y. Kwon and Y. Chae, "Traffic Adaptive IEEE 802.15.4 MAC for Wireless Sensor Networks, Embedded and Ubiquitous Computing", Lecture Notes in Computer Science Series, pp.864-873, Germany, 2006

13. J. Lee, et al., "ECAP: A Bursty Traffic Adaptation Algorithm for IEEE 802.15.4 BeaconEnabled Networks", Proceedings of the IEEE 65th Vehicular Technology Conference (VTC2007-Spring), pp. 203-207, Ireland, April 2007.

14. M. Neugebauer et al., "A new beacon order adaptation algorithm for IEEE 802.15.4 networks" Proceedings of the Second European Workshop on Wireless Sensor Networks, pp. 302-311, 2005.

15. M. Neugebauer et al., "Duty cycle adaptation with respect to traffic", Proceedings of the 10th IEEE Conference on Emerging Technologies and Factory Automation (ETFA 2005), pp.425-432, Italy, September 2005

16. OMNeT++. Available: http://www.omnetpp.org

17. InetManet FrameWork. Available: https://github.com/aarizaq/inetmanet-2.0.

18. Texas Instruments homepage. Available: http://www.ti.com/product/cc24 